\title{
CPU-Style SIMD Ray Traversal on GPUs Supplemental Material
}

\author{
Alexander Lier Marc Stamminger Kai Selgrad \\ Computer Graphics Group, University of Erlangen-Nuremberg
}

\begin{tabular}{|c|c|c|c|c|c|c|c|c|c|c|c|c|c|c|c|}
\hline Method & \multicolumn{3}{|c|}{ 2-Wide Reference } & \multicolumn{3}{|c|}{ 4-Wide Reference } & \multicolumn{3}{|c|}{ 2-Wide Ours } & \multicolumn{3}{|c|}{ 4-Wide Ours } & \multicolumn{3}{|c|}{ 8-Wide Ours } \\
\hline Ray type & prim. & diff. & diff. 8 & prim. & diff. 1 & diff. 8 & prim. & diff. 1 & diff. 8 & prim. & diff. 1 & diff. 8 & prim. & diff. 1 & diff. 8 \\
\hline Conference & 676.57 & 370.78 & 302.35 & 1.09 & 1.04 & 1.03 & 0.99 & 0.96 & 1.04 & 0.79 & 0.87 & 0.97 & 0.55 & 0.65 & 0.74 \\
\hline Fairy Forest & 408.95 & 263.81 & 61.25 & 1.00 & 1.00 & 1.01 & 0.92 & 1.00 & 1.16 & 0.76 & 0.92 & 1.26 & 0.56 & 0.71 & 1.12 \\
\hline Sibenik & 650.11 & 306.91 & 169.80 & 0.99 & 1.02 & 1.08 & 0.96 & 1.04 & 1.49 & 0.74 & 0.88 & 1.38 & 0.48 & 0.62 & 0.98 \\
\hline My Citadel & 366.05 & 195.66 & 95.81 & 1.10 & 1.03 & 1.03 & 1.16 & 1.25 & 1.27 & 0.77 & 0.91 & 1.05 & 0.55 & 0.75 & 0.94 \\
\hline Sponza & 439.54 & 219.99 & 129.37 & 1.03 & 1.02 & 1.02 & 0.92 & 1.08 & 1.47 & 0.78 & 0.96 & 1.47 & 0.48 & 0.65 & 1.06 \\
\hline Piper's Alley & 395.15 & 208.62 & 52.58 & 1.08 & 1.02 & 1.04 & 0.99 & 1.04 & 1.27 & 0.88 & 1.02 & 1.44 & 0.59 & 0.73 & 1.27 \\
\hline Hairball & 123.29 & 47.73 & 21.59 & 1.08 & 1.11 & 1.05 & 1.09 & 1.61 & 1.58 & 1.13 & 2.00 & 2.75 & 0.86 & 1.71 & 2.60 \\
\hline San Miguel & 231.12 & 90.81 & 37.24 & 1.06 & 1.03 & 1.01 & 1.05 & 1.49 & 1.72 & 0.92 & 1.49 & 2.51 & 0.63 & 1.13 & 2.14 \\
\hline Rungholt & 572.37 & 283.45 & 77.04 & 1.14 & 1.03 & 1.03 & 0.92 & 1.26 & 1.68 & 0.76 & 1.07 & 1.63 & 0.48 & 0.71 & 1.26 \\
\hline Powerplant & 289.60 & 161.45 & 83.23 & 1.07 & 1.00 & 1.03 & 1.12 & 1.32 & 1.80 & 0.92 & 1.37 & 2.25 & 0.64 & 1.05 & 1.84 \\
\hline Powerplant2 & 394.21 & 232.17 & 130.12 & 1.04 & 1.01 & 1.02 & 0.90 & 1.09 & 1.56 & 0.80 & 1.07 & 1.64 & 0.54 & 0.77 & 1.24 \\
\hline Average & 413.36 & 216.49 & 105.49 & 1.06 & 1.03 & 1.03 & 1.00 & 1.19 & 1.46 & 0.84 & 1.14 & 1.67 & 0.58 & 0.86 & 1.38 \\
\hline
\end{tabular}

Table 1: Absolute performance results in MRays/s for the 2-wide reference [Aila et al. 2012] utilizing triangle duplication according to the original implementation (first column). Results are segmented into primary, diffuse first bounce, and diffuse eighth bounce. The relative performance values of the 4-wide reference [Guthe 2014] and our approaches with 2-, 4-, and 8-wide BVHs are normalized to to the 2 -wide reference. 